\title{
Impact of Azimuth Ambiguities on Interferometric Performance
}

\author{
Michelangelo Villano, Student Member, IEEE, and Gerhard Krieger, Senior Member, IEEE
}

\begin{abstract}
The impact of azimuth ambiguities on interferometric performance in terms of phase bias and standard deviation of the interferometric phase is analyzed, resorting to the interferogram statistics for jointly circular Gaussian processes. The theoretical results are validated through simulation and compared with measurements on a TanDEM-X interferogram, affected by azimuth ambiguities.
\end{abstract}

Index Terms-Azimuth ambiguities, interferometry, microwave remote sensing, synthetic aperture radar (SAR).

\section{INTRODUCTION}

A ZIMUTH ambiguities arise in synthetic aperture radar (SAR) images from finite sampling of the Doppler spectrum at the pulse repetition frequency (PRF). Since the spectrum repeats at PRF intervals, the signal components outside this frequency interval fold back into the main part of the spectrum [1], [2].

The impact of azimuth ambiguities on interferometric performance is usually condensed in a coherence loss component $\gamma_{A m b, A z}[3]$, given by

$$
\gamma_{A m b, A z}=\frac{1}{1+A A S R}
$$

where $A A S R$ is the azimuth ambiguity-to-signal ratio. In this respect, azimuth ambiguities are considered in the same way as thermal noise. Furthermore, the increase in the AASR caused by an interferometric antenna beam misalignment and its impact on the standard deviation of the interferometric phase is considered in [4].

Fig. 1 displays a detail of a TanDEM-X [3] interferogram acquired over the Franz Josef Land, an archipelago located in the far north of Russia. The main acquisition parameters are given in Table I. In the top left-hand part, some sea ice, which surrounds the islands of the archipelago, can be distinguished. In this region, an unexpected coherence modulation can be observed, for which an explanation was not clear at the beginning. Taking a look to the interferometric phase, it can be noticed that the pattern of the bottom part of the image is somehow replicated in the sea ice region. The relative displacement of such a replica and the considerable backscatter difference between the

Manuscript received October 26, 2011; revised December 9, 2011 and January 27, 2012; accepted January 27, 2012.

The authors are with the Microwaves and Radar Institute, German Aerospace Center (DLR), 82234 Wessling, Germany (e-mail: michelangelo. villano@dlr.de; gerhard.krieger@dlr.de).

Color versions of one or more of the figures in this paper are available online at http://ieeexplore.ieee.org.

Digital Object Identifier 10.1109/LGRS.2012.2187271

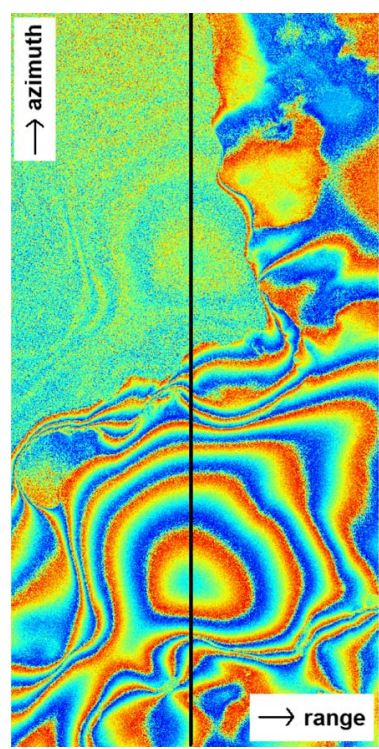

(a)

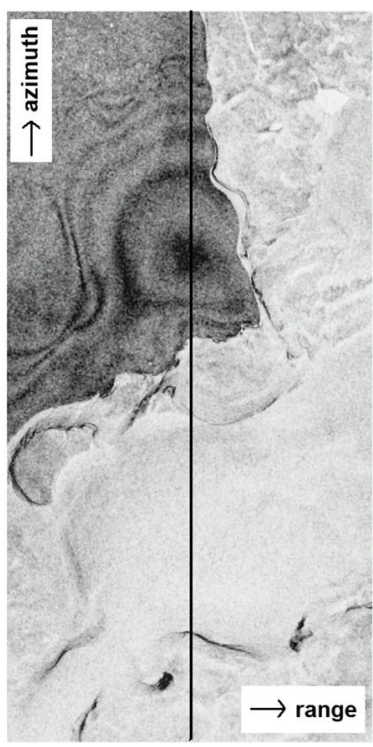

(b)
Fig. 1. Detail of a TanDEM-X interferogram affected by azimuth ambiguities, acquired over the Franz Josef Land, Russia (approximately $2.8 \mathrm{~km} \times 9.2 \mathrm{~km}$ ). (a) Interferometric phase. (b) Magnitude of the complex coherence. The vertical solid lines have been added to highlight the azimuth cut to which Figs. 5 and 6 refer.

TABLE I

MAIN ACQUisition PARAMETERS FOR THE TanDEM-X INTERFEROGRAM OF FIG. 1

\begin{tabular}{|l|l|}
\hline Location (latitude, longitude) & $80.3^{\circ} \mathrm{N}, 54.5^{\circ} \mathrm{E}$ \\
\hline Date and time & $30 / 12 / 2010,12: 41: 33$ \\
\hline Chirp bandwidth & $100 \mathrm{MHz}$ \\
\hline PRF & $3113 \mathrm{~Hz}$ \\
\hline Processed Doppler bandwidth & $2765 \mathrm{~Hz}$ \\
\hline Doppler centroid & $<25.6 \mathrm{~Hz}$ \\
\hline Height of ambiguity & $52.4 \mathrm{~m}$ \\
\hline Effective along-track baseline & $18.7 \mathrm{~m}$ \\
\hline
\end{tabular}

two areas suggest that this effect may be due to azimuth ambiguities. Therefore, not only are azimuth ambiguities responsible for a coherence loss component, but they could also determine a significant phase bias, as well as a coherence modulation.

A much deeper analysis of this phenomenon may be conducted by modeling the interferogram affected by azimuth ambiguities as the sum of interfering components and deriving its statistics. 


\section{Simple Model For InTERFEROGRAms AfFected by Azimuth Ambiguities}

Let $u[x, y]$ be a single-look complex SAR image. $u[x, y]$ can be written as the sum of an ambiguity-free signal $m[x, y]$, from now on referred to as main signal, and a signal due to the azimuth ambiguity $a[x, y]$, from now on referred to as ambiguity signal

$$
u[x, y]=m[x, y]+a[x, y] .
$$

We assume that the in-phase and quadrature components of $m[x, y]$ are independent identically distributed Gaussian random variables with mean zero and variance $P_{m} / 2$, where $P_{m}$ is the variance or power of $m[x, y]$. We also assume that the in-phase and quadrature components of $a[x, y]$ are independent identically distributed Gaussian random variables with mean zero and variance $P_{a} / 2$, where $P_{a}$ is the variance or power of $a[x, y]$

$$
\begin{aligned}
& P_{m}=E\left\{|m[x, y]|^{2}\right\} \\
& P_{a}=E\left\{|a[x, y]|^{2}\right\} .
\end{aligned}
$$

Furthermore, we assume that $m[x, y]$ and $a[x, y]$ are statistically independent. As a consequence, the in-phase and quadrature components of $u[x, y]$ are independent identically distributed Gaussian random variables with mean zero and variance $\left(P_{m}+P_{a}\right) / 2$, where $P_{m}+P_{a}$ is the variance or power of $u[x, y]$.

In an interferometric scenario, both master and slave images, $u_{1}[x, y]$ and $u_{2}[x, y]$, are affected by azimuth ambiguities

$$
\begin{aligned}
& u_{1}[x, y]=m_{1}[x, y]+a_{1}[x, y] \\
& u_{2}[x, y]=m_{2}[x, y]+a_{2}[x, y] .
\end{aligned}
$$

$m_{1}[x, y]$ and $m_{2}[x, y]$ are characterized by a variance or power equal to $P_{m}$ and are in general correlated, being their complex correlation coefficient $\gamma_{m}=\left|\gamma_{m}\right| \exp \left(j \phi_{0 m}\right) . a_{1}[x, y]$ and $a_{2}[x, y]$ are characterized by a variance or power equal to $P_{a}$ and are also in general correlated, being their complex correlation coefficient $\gamma_{a}=\left|\gamma_{a}\right| \exp \left(j \phi_{0 a}\right)$. $m_{k}[x, y], k=1,2$, and $a_{k}[x, y], k=1,2$, are instead uncorrelated.

If an interferogram is formed from $u_{1}[x, y]$ and $u_{2}[x, y]$, four components, denoted as $v_{1}[x, y], v_{2}[x, y], v_{3}[x, y]$, and $v_{4}[x, y]$, arise

$$
\begin{aligned}
v[x, y]= & u_{1}[x, y] u_{2}^{*}[x, y] \\
= & \left(m_{1}[x, y]+a_{1}[x, y]\right)\left(m_{2}[x, y]+a_{2}[x, y]\right)^{*} \\
= & m_{1}[x, y] m_{2}^{*}[x, y]+m_{1}[x, y] a_{2}^{*}[x, y] \\
& +a_{1}[x, y] m_{2}^{*}[x, y]+a_{1}[x, y] a_{2}^{*}[x, y] \\
= & v_{1}[x, y]+v_{2}[x, y]+v_{3}[x, y]+v_{4}[x, y] .
\end{aligned}
$$

$v_{1}[x, y]$ is the interferometric contribution formed from the two main signals, $m_{1}[x, y]$ and $m_{2}[x, y], v_{4}[x, y]$ is the interferometric contribution formed from the two ambiguity signals, $a_{1}[x, y]$ and $a_{2}[x, y]$, while $v_{2}[x, y]$ and $v_{3}[x, y]$ are the interferometric contributions obtained by combining the main and ambiguity signals.

\section{Statistical CharaCteris ATION OF INTERFEROGRAMS AFFECTED BY AZIMUTH AMbiguities}

The phase bias and the standard deviation of the interferometric phase of an interferogram affected by azimuth ambiguities are analytically derived in the following. The expression of the joint probability density function (PDF) of magnitude and phase of the interferogram is recognized based on some considerations on jointly circular Gaussian processes, while its parameters are obtained by equating the expression of the expected value of the complex interferogram with the sum of the expected values of the four components of (5).

The processes $m_{1}[x, y]$ and $m_{2}[x, y]$ can be assumed to be jointly circular Gaussian. The processes $a_{1}[x, y]$ and $a_{2}[x, y]$ can also be assumed to be jointly circular Gaussian. Being $\mathbf{u}[x, y]=\left[u_{1}[x, y], u_{2}[x, y]\right]$ a linear functional of jointly circular Gaussian processes, namely the sum of $\mathbf{m}[x, y]=$ $\left[m_{1}[x, y], m_{2}[x, y]\right]$ and $\mathbf{a}[x, y]=\left[a_{1}[x, y], a_{2}[x, y]\right], u_{1}[x, y]$ and $u_{2}[x, y]$ are also jointly circular Gaussian [5].

The statistics of SAR interferograms are discussed in [6] and [7]. Being $u_{1}[x, y]$ and $u_{2}[x, y]$ jointly circular Gaussian processes, the interferogram $v[x, y]$ can be statistically characterized in terms of a joint PDF of magnitude $|v|$ and phase $\phi[7]$

$$
\begin{aligned}
p_{|v|, \phi}(|v|, \phi)=\frac{2|v|}{\pi I^{2}\left(1-|\gamma|^{2}\right)} \exp & \left\{\frac{2|\gamma||v| \cos \left(\phi-\phi_{0}\right)}{I\left(1-|\gamma|^{2}\right)}\right\} \\
& \times K_{0}\left(\frac{2|v|}{I\left(1-|\gamma|^{2}\right)}\right)
\end{aligned}
$$

where $K_{0}(\cdot)$ is the modified Bessel function of order zero.

This joint PDF is characterized by three parameters, namely $I$, the geometric mean of the powers of the two complex processes $u_{1}[x, y]$ and $u_{2}[x, y],|\gamma|$, the magnitude of the complex coherence of the interferogram formed from $u_{1}[x, y]$ and $u_{2}[x, y]$, and $\phi_{0}$, the expected value of the interferometric phase of the interferogram formed from $u_{1}[x, y]$ and $u_{2}[x, y]$. The geometric mean of the powers $I$ can be straightforwardly evaluated. As the powers of $u_{1}[x, y]$ and $u_{2}[x, y]$ are both equal to $P_{m}+P_{a}$, it holds

$$
I=P_{m}+P_{a} .
$$

The remaining two parameters, $|\gamma|$ and $\phi_{0}$, will be determined in the following.

The expected value of $v[x, y]$ can be evaluated by summing the expected values of its four components. The expected values of $v_{2}[x, y]$ and $v_{3}[x, y]$ are equal to zero, as $v_{2}[x, y]$ and $v_{3}[x, y]$ are products of uncorrelated random variables. The complex expected value $E\{v[x, y]\}$ is then given by

$$
\begin{aligned}
E\{\{[x, y]\} & \\
= & E\left\{v_{1}[x, y]\right\}+E\left\{v_{2}[x, y]\right\} \\
& +E\left\{v_{3}[x, y]\right\}+E\left\{v_{4}[x, y]\right\} \\
= & E\left\{v_{1}[x, y]\right\}+E\left\{v_{4}[x, y]\right\} \\
= & P_{m}\left|\gamma_{m}\right| \exp \left(j \phi_{0 m}\right)+P_{a}\left|\gamma_{a}\right| \exp \left(j \phi_{0 a}\right) \\
= & \left|P_{m}\right| \gamma_{m}\left|\exp \left(j \phi_{0 m}\right)+P_{a}\right| \gamma_{a}\left|\exp \left(j \phi_{0 a}\right)\right| \\
& \exp \left(j \arg \left\{P_{m}\left|\gamma_{m}\right| \exp \left(j \phi_{0 m}\right)+P_{a}\left|\gamma_{a}\right| \exp \left(j \phi_{0 a}\right)\right\}\right) \\
= & P_{m} \sqrt{\left|\gamma_{m}\right|^{2}+\left(\frac{P_{a}}{P_{m}}\right)^{2}\left|\gamma_{a}\right|^{2}+2 \frac{P_{a}}{P_{m}}\left|\gamma_{m}\right|\left|\gamma_{a}\right| \cos \left(\phi_{0 a}-\phi_{0 m}\right)} \\
& \exp \left(j \arg \left\{\exp \left(j \phi_{0 m}\right)+\frac{P_{a}}{P_{m}} \frac{\left|\gamma_{a}\right|}{\left|\gamma_{m}\right|} \exp \left(j \phi_{0 a}\right)\right\}\right) . \text { (8) }
\end{aligned}
$$



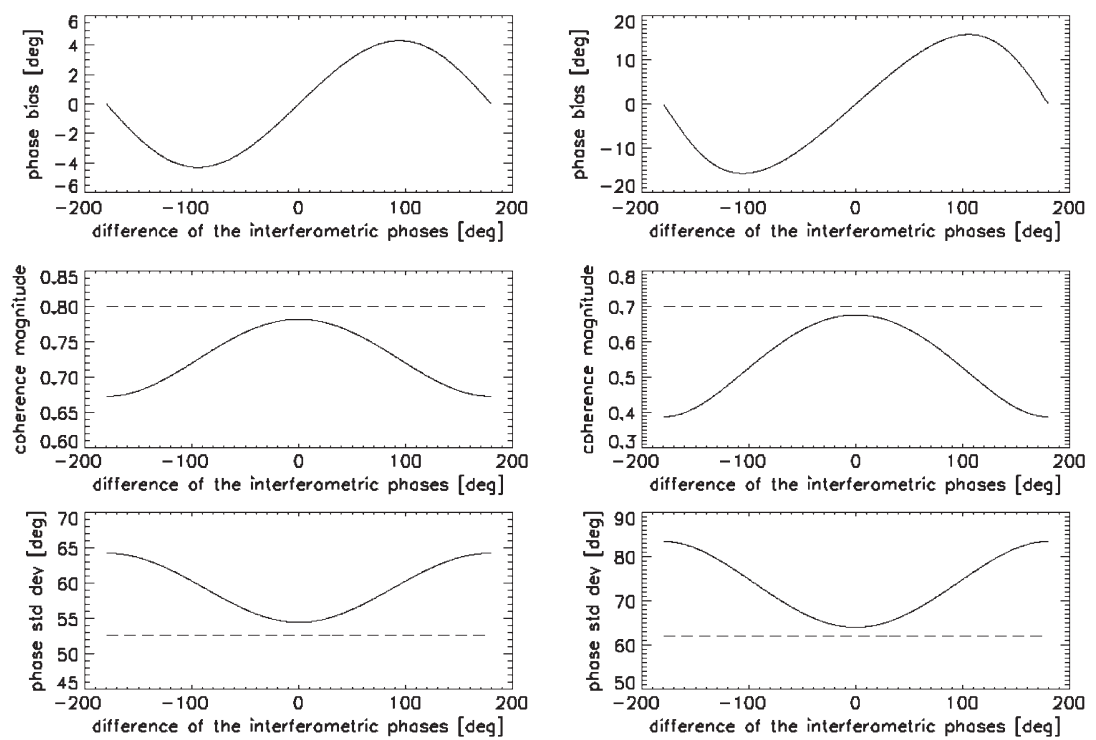

(a)

(b)
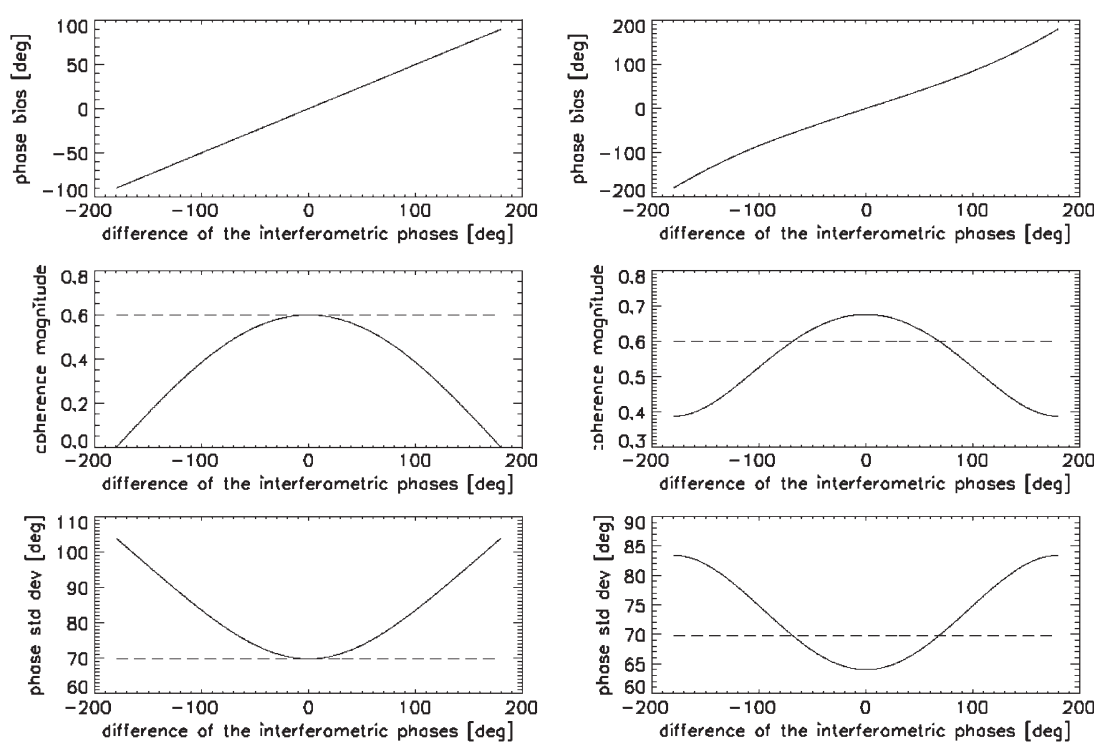

(c)

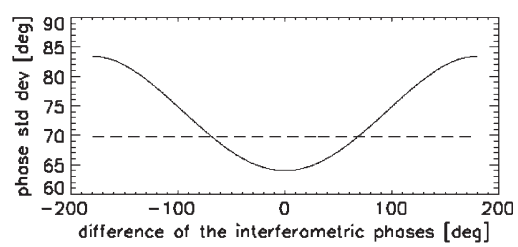

(d)

Fig. 2. Phase bias, coherence magnitude, and standard deviation of the interferometric phase as a function of the difference of the interferometric phases $\phi_{0 a}-\phi_{0 m}$, for different sets of parameters. The dashed lines represent the magnitude of the complex coherence and the standard deviation of the interferometric phase in the absence of ambiguities. (a) $P_{a} / P_{m}=-10 \mathrm{~dB},\left|\gamma_{m}\right|=0.8,\left|\gamma_{a}\right|=0.6$. (b) $P_{a} / P_{m}=-5 \mathrm{~dB},\left|\gamma_{m}\right|=0.7,\left|\gamma_{a}\right|=0.6$. (c) $P_{a} / P_{m}=0 \mathrm{~dB}$, $\left|\gamma_{m}\right|=0.6,\left|\gamma_{a}\right|=0.6$. (d) $P_{a} / P_{m}=5 \mathrm{~dB},\left|\gamma_{m}\right|=0.6,\left|\gamma_{a}\right|=0.7$.

The expected value of $v[x, y]$ can also be expressed as a function of the parameters of the joint PDF of (6) as

$$
E\{v[x, y]\}=I|\gamma| \exp \left(j \phi_{0}\right)=\left(P_{m}+P_{a}\right)|\gamma| \exp \left(j \phi_{0}\right) .
$$

The parameters $|\gamma|$ and $\phi_{0}$ can therefore be obtained by equating the amplitudes and phases of the expressions given in (8) and (9), holding

$|\gamma|=\frac{1}{1+\frac{P_{a}}{P_{m}}} \sqrt{\left|\gamma_{m}\right|^{2}+\left(\frac{P_{a}}{P_{m}}\right)^{2}\left|\gamma_{a}\right|^{2}+2 \frac{P_{a}}{P_{m}}\left|\gamma_{m}\right|\left|\gamma_{a}\right| \cos \left(\phi_{0 a}-\phi_{0 m}\right)}$

$\phi_{0}=\arg \left\{\exp \left(j \phi_{0 m}\right)+\frac{P_{a}}{P_{m}} \frac{\left|\gamma_{a}\right|}{\left|\gamma_{m}\right|} \exp \left(j \phi_{0 a}\right)\right\}$

respectively. It is interesting to notice that, for $\left|\gamma_{a}\right|=0$, azimuth ambiguities are only responsible for a coherence loss component, exactly the one given in (1).
The phase bias resulting from the presence of the azimuth ambiguity is thus given by

$$
\begin{aligned}
\phi_{\text {bias }}=\phi_{0}- & \phi_{0 m}=\arg \left\{1+\frac{P_{a}}{P_{m}} \frac{\left|\gamma_{a}\right|}{\left|\gamma_{m}\right|} \exp \left(j\left(\phi_{0 a}-\phi_{0 m}\right)\right)\right\} \\
=\arg & \left\{1+\frac{P_{a}}{P_{m}} \frac{\left|\gamma_{a}\right|}{\left|\gamma_{m}\right|} \cos \left(\phi_{0 a}-\phi_{0 m}\right)\right. \\
& \left.+j \frac{P_{a}}{P_{m}} \frac{\left|\gamma_{a}\right|}{\left|\gamma_{m}\right|} \sin \left(\phi_{0 a}-\phi_{0 m}\right)\right\} .
\end{aligned}
$$

The phase variance instead can be related to the magnitude of the complex coherence $|\gamma|$ from (10) by the following formula [7]:

$$
\sigma_{\phi}=\sqrt{\frac{\pi^{2}}{3}-\pi \arcsin (|\gamma|)+\arcsin ^{2}(|\gamma|)-\frac{\operatorname{Li}_{2}\left(|\gamma|^{2}\right)}{2}}
$$

where $\mathrm{Li}_{2}(\cdot)$ is Euler's dilogarithm. 


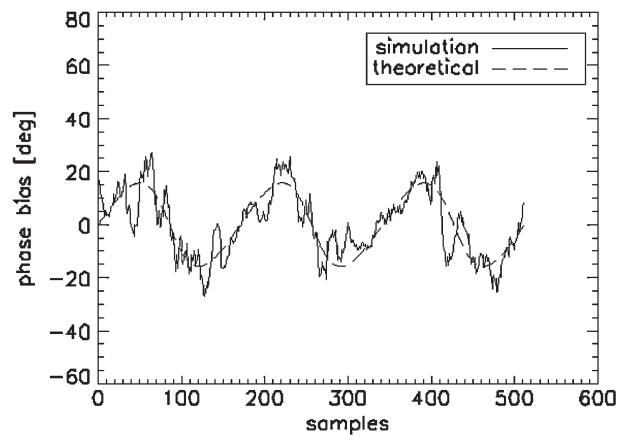

(a)

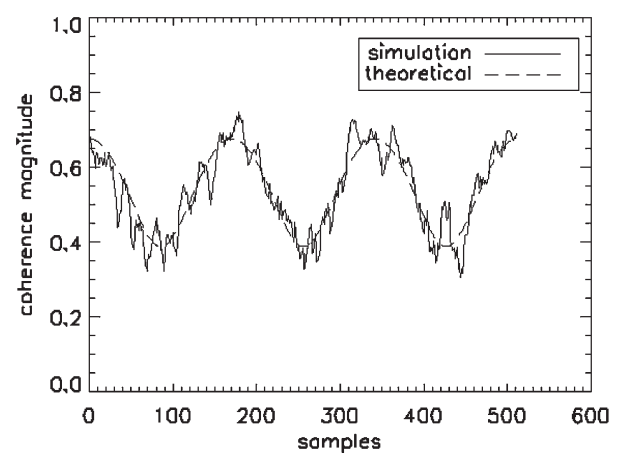

(b)

Fig. 3. Comparison of simulated and theoretical results. The interferometric phases of the interferogram formed from the main signals and the one formed from the ambiguity signals are constant and linear, respectively. $P_{a} / P_{m}=$ $-5 \mathrm{~dB},\left|\gamma_{m}\right|=0.7,\left|\gamma_{a}\right|=0.6$. (a) Phase bias. (b) Magnitude of the complex coherence.

Fig. 2 shows the phase bias, the magnitude of the complex coherence, and the standard deviation of the interferometric phase, as a function of the difference of the interferometric phases $\phi_{0 a}-\phi_{0 m}$, for different values of $P_{a} / P_{m},\left|\gamma_{m}\right|$, and $\left|\gamma_{a}\right|$.

The theoretical results have also been validated through simulation. An interferogram affected by the ambiguity has been generated according to the model of (4) and (5), the interferometric phases of the interferograms $v_{1}[x, y]$ and $v_{4}[x, y]$ being constant and linear, respectively. $P_{a} / P_{m},\left|\gamma_{m}\right|$, and $\left|\gamma_{a}\right|$ have been set to $-5 \mathrm{~dB}, 0.7$, and 0.6 , respectively. The theoretical phase bias and magnitude of the complex coherence are superimposed on the simulated ones in Fig. 3, and are in good agreement.

\section{Magnitude of the Complex Coherence OF THE INTERFEROMETRIC CONTRIBUTION Formed From the Ambiguity Signals}

In the following, an expression for the magnitude of the complex coherence of the interferometric contribution formed from the ambiguity signals $\left|\gamma_{a}\right|$ is derived.

Assuming that the right or the left azimuth ambiguity predominates over the other one, for each area of the interferogram affected by the ambiguity, an area responsible for the ambiguity itself can be identified. Let us denote as $\left|\gamma_{a R}\right|$ the magnitude of the complex coherence of the area responsible for the ambiguity. The magnitudes of the complex coherences $\left|\gamma_{a}\right|$ and $\left|\gamma_{a R}\right|$ are equal but for the coherence loss component due to the signal-to-noise ratio (SNR). Moreover, the SNR of the ambiguity signal and the SNR of the signal responsible for the ambiguity are related through the first azimuth ambiguity-tosignal ratio (FAASR), defined as in

$$
F A A S R=\frac{\int_{-B_{p} / 2}^{B_{p} / 2} G^{2}(f+P R F) H^{2}(f) d f}{\int_{-B_{p} / 2}^{B_{p} / 2} G^{2}(f) H^{2}(f) d f}
$$

where $B_{p}$ is the processed Doppler bandwidth, $G^{2}(f)$ is the two-way antenna power pattern in azimuth, $H(f)$ accounts for the amplitude weighting of the Doppler spectrum applied in the processing, and where uniform scene reflectivity is assumed.

The FAASR must not be confused with the AASR. If a scene is considered, where only a single point target is present, the SAR image corresponding to this scene will include a main response and many infinite right and left ambiguities. Always assuming uniform scene reflectivity, while the AASR is the ratio of the power of all ambiguities to the power of the main response, the FAASR is the ratio of the power of the first-order right or left ambiguity to the power of the main response.

Denoting as $S N R_{R}$ the SNR of the area responsible for the ambiguity, it holds

$$
\begin{aligned}
\left|\gamma_{a}\right| & =\left|\gamma_{a R}\right| \frac{\frac{1}{1+S N R_{R}^{-1} F A A S R^{-1}}}{\frac{1}{1+S N R_{R}^{-1}}} \\
& =\left|\gamma_{a R}\right| \frac{1+S N R_{R}}{F A A S R^{-1}+S N R_{R}}
\end{aligned}
$$

\section{COMPARISON With MEASUREMENTS ON REAL SAR INTERFEROGRAMS}

An ambiguity-free interferogram can be obtained by removing azimuth ambiguities in both the master and slave images by means of a Wiener filter [8]. This technique has been applied to several interferograms affected by azimuth ambiguities, showing its effectiveness for both point-like and distributed scatterers. The removal is achieved at the expense of a slight degradation of the azimuth resolution, which only interests the areas affected by ambiguities.

The ambiguity-free version of the interferogram of Fig. 1 is provided in Fig. 4. An azimuth cut, highlighted in Figs. 1 and 4 by the vertical solid lines, is analyzed. Fig. 5 shows the estimated phase bias, obtained by taking the difference of the interferometric phases of the interferogram affected by ambiguities and the ambiguity-free one, while Fig. 6 shows the magnitude of the complex coherence for the two mentioned interferograms.

In order to show that the observed phase bias and the magnitude of the complex coherence are consistent with their theoretical expressions, given in (12) and (10), respectively, $P_{a} / P_{m}$, $\left|\gamma_{m}\right|$, and $\left|\gamma_{a}\right|$ have to be estimated for the images under analysis. The ratio $P_{a} / P_{m}$ can be estimated as explained in [8]. In the areas of the image where the effects of ambiguities are particularly visible, this ratio is approximately equal to $0 \mathrm{~dB}$. $\left|\gamma_{m}\right|$ can be retrieved from the magnitude of the complex coherence of the ambiguity-free interferogram (right portion of solid line in Fig. 6) and is equal to $\left|\gamma_{m}\right|=0.45 .\left|\gamma_{a}\right|$ can be instead estimated using (15), where $\left|\gamma_{a R}\right|=0.88$ (left portion of solid line in Fig. 6), $S N R_{R}=23.5 \mathrm{~dB}$ (from system performance analysis), and $F A A S R=-22.73 \mathrm{~dB}$, obtaining $\left|\gamma_{a}\right|=0.48$. 


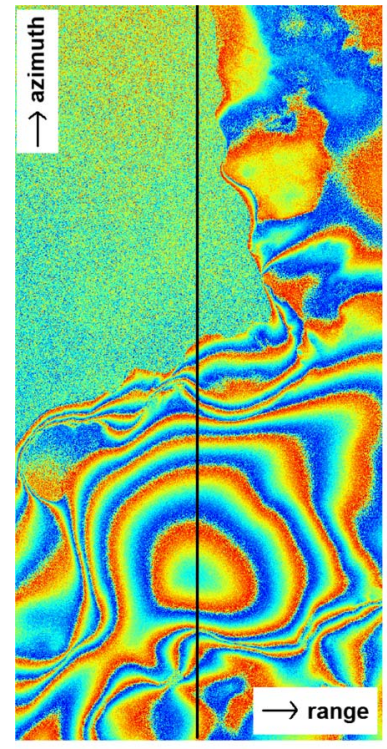

(a)

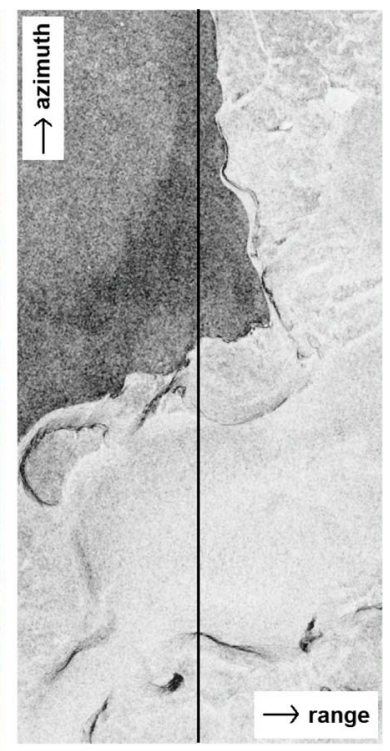

(b)
Fig. 4. Ambiguity-free version of the interferogram of Fig. 1. (a) Interferometric phase. (b) Magnitude of the complex coherence. The vertical solid lines have been added to highlight the azimuth cut to which Figs. 5 and 6 refer.

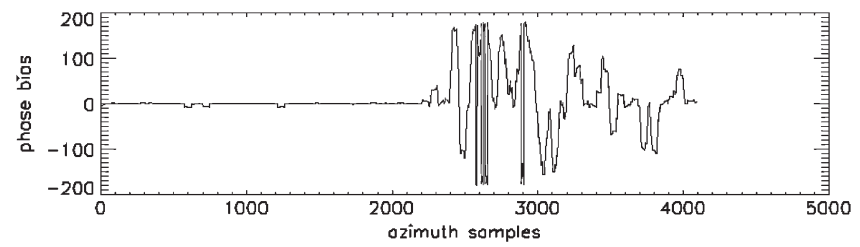

Fig. 5. Estimated phase bias for the azimuth cut highlighted by the solid line in Figs. 1 and 4.

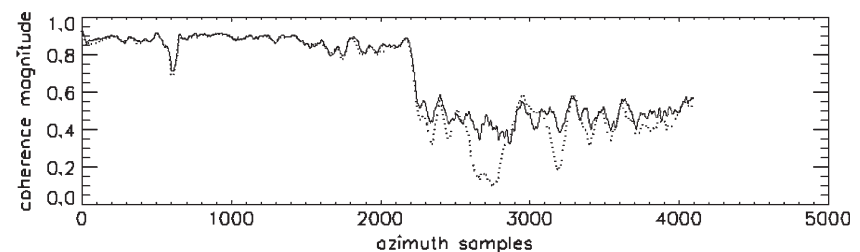

Fig. 6. Magnitude of the complex coherence for the azimuth cut highlighted by the solid line in Fig. 1 (dotted) and Fig. 4 (solid).

Fig. 7 shows the phase bias, the magnitude of the complex coherence, and the standard deviation of the interferometric phase as a function of $\phi_{0 a}-\phi_{0 m}$, for such values of $P_{a} / P_{m}$, $\left|\gamma_{m}\right|$, and $\left|\gamma_{a}\right|$. It can be noticed that the predicted range of phase bias is consistent with Fig. 5, as well as the predicted range of the magnitude of the complex coherence is consistent with the dotted line in Fig. 6.

\section{CONCLUSION}

Azimuth ambiguities affect the interferometric performance, modifying the interferogram statistics. As a consequence, a phase bias, dependent on the difference of the interferometric
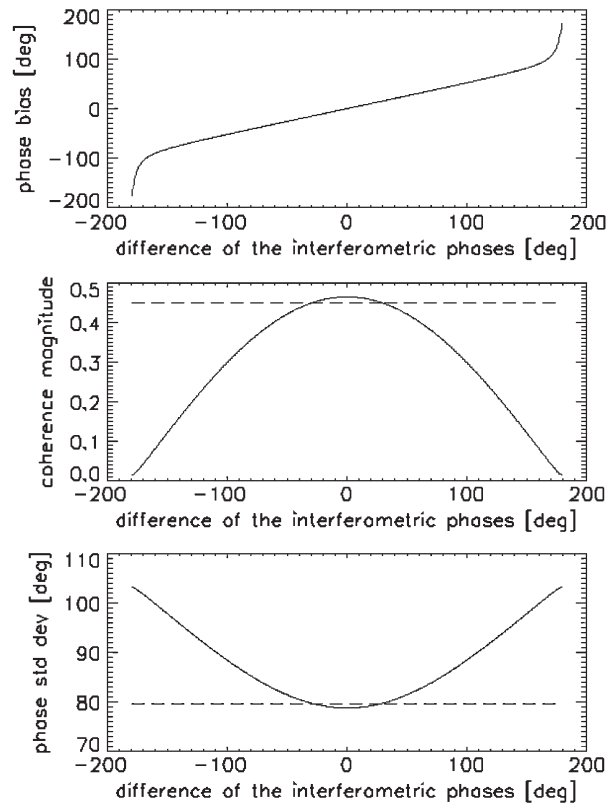

Fig. 7. Phase bias, magnitude of the complex coherence, and standard deviation of the interferometric phase as a function of the difference of the interferometric phases $\phi_{0 a}-\phi_{0 m}$, for different sets of parameters. The dashed lines represent the magnitude of the complex coherence and the standard deviation of the interferometric phase in the absence of ambiguities. $P_{a} / P_{m}=$ $0 \mathrm{~dB},\left|\gamma_{m}\right|=0.45,\left|\gamma_{a}\right|=0.48$.

phases of the interferogram of ambiguity and main signals, is introduced. As far as the magnitude of the complex coherence is concerned, it decreases as the absolute value of the difference of the interferometric phases increases. For high values of $P_{a} / P_{m}$, the coherence can also exceed the magnitude of the complex coherence in the absence of ambiguities. This behavior is consistent with observations on real SAR interferograms.

\section{REFERENCES}

[1] F. K. Li and W. T. K. Johnson, "Ambiguities in spaceborne synthetic aperture radar systems," IEEE Trans. Aerosp. Electron. Syst., vol. 19, no. 3, pp. 389-397, May 1983.

[2] A. Freeman, W. T. K. Johnson, B. Huneycutt, R. Jordan, S. Hensley, P. Siqueira, and J. Curlander, "The "Myth" of the minimum SAR antenna area constraint," IEEE Trans. Geosci. Remote Sens., vol. 38, no. 1, pp. 320324, Jan. 2000

[3] G. Krieger, A. Moreira, H. Fiedler, I. Hajnsek, M. Werner, M. Younis, and M. Zink, "TanDEM-X: A satellite formation for high-resolution SAR interferometry," IEEE Trans. Geosci. Remote Sens., vol. 45, no. 11, pp. 33173341, Nov. 2007.

[4] D. Geudtner, M. Zink, C. Gierull, and S. Shaffer, "Interferometric alignment of the X-SAR antenna system on the space shuttle radar topography mission," IEEE Trans. Geosci. Remote Sens., vol. 40, no. 5, pp. 995-1006, May 2002.

[5] R. G. Gallager, Circularly-Symmetric Gaussian Random Vectors. [Online]. Available: http://www.rle.mit.edu/rgallager/documents/CircSymGauss.pdf

[6] D. Just and R. Bamler, "Phase statistics of interferograms with applications to synthetic aperture radar," Appl. Opt., vol. 33, no. 20, pp. 4361-4368, 1994.

[7] R. Bamler and P. Hartl, "Synthetic aperture radar interferometry," Inv. Probl., vol. 14, no. 4, pp. R1-R54, Feb. 1998.

[8] A. Monti Guarnieri, "Adaptive removal of azimuth ambiguities in SAR images," IEEE Trans. Geosci. Remote Sens., vol. 43, no. 3, pp. 625-633, Mar. 2005. 